\title{
cAMP Response Element-Binding Protein 1 Feedback Loop Is Necessary for Consolidation of Long-Term Synaptic Facilitation in Aplysia
}

\author{
Rong-Yu Liu, ${ }^{\star}$ Diasinou Fioravante, ${ }^{\star}$ Shreyansh Shah, and John H. Byrne \\ Department of Neurobiology and Anatomy, W. M. Keck Center for the Neurobiology of Learning and Memory, The University of Texas Medical School at \\ Houston, Houston, Texas 77030
}

\begin{abstract}
The transcription factor cAMP response element (CRE)-binding protein (CREB) plays an essential role in the induction of many forms of long-term synaptic plasticity. Levels of CREB1, the Aplysia homolog of CREB, show sustained elevations for several hours after the induction of long-term synaptic facilitation (LTF). Furthermore, CREB1 binds to the promoter of its own gene. These results suggest the existence of a CREB1-positive feedback loop that contributes to the consolidation of LTF. In the present study, we provide a detailed, quantitative characterization of the dynamics of CREB1 mRNA and protein as well as CREB1 phosphorylation after LTF induction. Injections of CRE oligonucleotides prevented the increase in CREB1 in response to 5-HT, corroborating the existence of the CREB1 feedback loop. This loop probably sustains CRE-dependent gene transcription, which remains elevated for at least $12 \mathrm{~h}$ after LTF induction. LTF is blocked by injection of CREB1 antibody after the induction phase, suggesting that the CREB1-positive feedback is required for consolidation of LTF.
\end{abstract}

Key words: CREB; synaptic plasticity; memory consolidation; long-term facilitation; serotonin

\section{Introduction}

Long-term memory and plasticity, including long-term synaptic facilitation (LTF) of the Aplysia sensorimotor synapse, depend on the activation of transcription factors that regulate genes necessary for synaptic plasticity. Recently, it has become clear that among the genes regulated by transcription factors are those coding for transcription factors themselves. One such gene is creb1 (Mohamed et al., 2005), the Aplysia homolog of mammalian creb (Bartsch et al., 1998). The neuromodulator serotonin (5-HT), which is released in response to sensitizing stimuli (Levenson et al., 1999; Marinesco and Carew, 2002), leads to phosphorylation and activation of cAMP response element (CRE)-binding protein 1 (CREB1) through activation of protein kinase A (PKA) (Bartsch et al., 1998; Müller and Carew, 1998). CREB1-mediated gene expression is required for induction of LTF (Dash et al., 1990; Bartsch et al., 1998). However, the requirement for gene expression is not limited to the induction phase. The necessity of prolonged transcription and translation persists for at least 7-9 h after induction (Alberini et al., 1994; O'Leary et al., 1995).

Previous studies (Bartsch et al., 1998) showed that levels of

Received Feb. 5, 2007; revised Jan. 7, 2008; accepted Jan. 9, 2008.

This work was supported by National Institutes of Health Grant NS019895. We thank E. Kartikaningrum and J. Liu for preparing the cultures, W. Yao for assistance with the treatment procedures, L. Cleary and P. Smolen for comments on this manuscript, and B. Kaang for providing plasmids.

${ }^{*}$ R.-Y.L. and D.F. contributed equally to this work.

Correspondence should be addressed to Dr. John H. Byrne, Department of Neurobiology and Anatomy, W. M. Keck Center for the Neurobiology of Learning and Memory, The University of Texas Medical School at Houston, Houston, TX 77030. E-mail: John.H.Byrne@uth.tmc.edu.

D0I:10.1523/JNEUROSCI.3848-07.2008

Copyright $\odot 2008$ Society for Neuroscience $\quad$ 0270-6474/08/281970-07\$15.00/0
CREB1 increased after whole-animal exposure to solutions containing 5-HT, suggesting that CREB1 may be regulated during LTF. We previously extended these findings by discovering a CRE in the promoter region of the creb1 gene (Mohamed et al., 2005). Moreover, treatment of ganglia with five pulses of 5-HT, a protocol commonly used to induce LTF (Montarolo et al., 1986; Mauelshagen et al., 1998), resulted in enhanced recruitment of CREB1 to its promoter (Mohamed et al., 2005). These observations suggested that CREB1 can regulate its own expression, giving rise to a CREB1-positive feedback loop. The presence of such a loop could result in a prolonged increase in crebl expression and a consequent increase in CREB1-mediated transcription, which could extend beyond LTF induction. The goal of the present study was to characterize the time course of expression of creb1 after 5-HT treatment and to investigate the functional significance of the CREB1 feedback loop for the consolidation of LTF.

\section{Materials and Methods}

Tissue preparation and pharmacological treatment. For each measurement of mRNA, two naive animals were anesthetized by injection of isotonic $\mathrm{MgCl}_{2}(0.5 \mathrm{ml} / \mathrm{g})$, and the two bilaterally symmetric pairs of pleuralpedal ganglia were surgically isolated. To obtain sufficient RNA for quantitative reverse transcription PCR (qRT-PCR), a ganglion from the right side of one animal and one from the left side of the second animal were grouped together. The two remaining ganglia formed a second group. Each group was then randomly assigned to receive either a control (vehicle) or a 5-HT treatment. For Western blot analysis, the left and right pleural-pedal ganglia from one animal were randomly assigned to receive either a control (vehicle) or a 5-HT treatment. After trimming of the connective tissue sheath in isotonic $\mathrm{MgCl}_{2}$ :artificial seawater (ASW; 1:1 
$\mathrm{v} / \mathrm{v})$, ganglia in both groups were rinsed with modified L15:ASW (1:1 v/v) and rested at $18^{\circ} \mathrm{C}$ for $1-2 \mathrm{~h}$. Subsequently, groups were treated with five 5 min pulses of either vehicle (L15:ASW) or $50 \mu \mathrm{M} 5$-HT with an interpulse interval of $20 \mathrm{~min}$. Either immediately or at the indicated times after treatment, ganglia were rinsed with L15:ASW, rapidly frozen on dry ice, and stored at $-80^{\circ} \mathrm{C}$ until further processing. The composition of ASW and modified L15 has been published previously (Zhang et al., 1997; Antzoulatos et al., 2003).

$q R T-P C R$ analysis of CREB1 mRNA. Aplysia pleural ganglia were treated with five 5 min pulses of either vehicle (L15:ASW) or $50 \mu \mathrm{M} 5$-HT with an interpulse interval of $20 \mathrm{~min}$. Total RNA was isolated from frozen pleural ganglia using Trizol (Invitrogen, Carlsbad, CA) and treated with RNase-free DNase I to remove any contaminating genomic DNA. Quantification of mRNA was done by qRT-PCR as described previously (Mohamed et al., 2005).

Western blots. Pleural-pedal ganglia were lysed at various time points $(0,1,2,5,12$, and $24 \mathrm{~h})$ after the end of treatment with vehicle or 5 -HT in ice-cold buffer containing the following (in mM): 10 EDTA, 20 Tris, $\mathrm{pH}$ $7.5,1 \mathrm{Na}$ orthovanadate, $1 \mathrm{DTT}, 2 \mathrm{NaF}, 2 \mathrm{NaPPi}, 0.5$ okadaic acid, 1 PMSF, $1 \%$ SDS, $1 \%$ protease inhibitor cocktail (Sigma, St. Louis, MO) and stored at $-20^{\circ} \mathrm{C}$ until further use. Thirty micrograms of Aplysia pleural-pedal extract from each group were resolved using SDS-PAGE. Protein samples were transferred to a nitrocellulose membrane. The membrane was probed with anti-phospho-ApCREB1 (anti-pCREB1; 1:1000) followed by incubation with peroxidase-conjugated secondary antibody (1:2000; Zymed, San Francisco, CA). Immunoreactive bands were visualized by ECL (GE Healthcare, Pittsburgh, PA) and analyzed with ImageQuant 5.0 software. The same membrane was stripped and reprobed with anti-total ApCREB1 (anti-tCREB1) antibody (1:500). The anti-tCREB1 antibodies were raised by a commercial vendor (Genemed Synthesis, South San Francisco, CA) against the Ser ${ }^{85}$-phosphorylated and the unphosphorylated versions of the bovine serum albuminconjugated CREB1 peptide KKRREILTRRPSYRK (Mohamed et al., 2005). An antibody to glyceraldehyde-3-phosphate dehydrogenase (GAPDH) (1:2000; IMGENEX, San Diego, CA) was used to provide a loading control. Immunoreactive bands were visualized by ECL and analyzed with ImageQuant 5.0 software.

Antibody injections and immunofluorescence analysis. Rabbit IgG or anti-tCREB1 antibody, which was also used in Western blots and immunofluorescence analyses, was injected at $0.5 \mu \mathrm{g} / \mu \mathrm{l}$ in $100 \mathrm{~mm} \mathrm{KCl}-0.1 \%$ injection dye (Alexa 488-10 kDa dextran or Fluorescein-70 kDa dextran). Sensory neurons (SNs) were injected either $2 \mathrm{~h}$ before or $1 \mathrm{~h}$ after 5-HT treatment, as indicated. At various time points after the end of treatment, cells were processed for immunofluorescence analysis using a Cy3-conjugated secondary antibody, as described by Chin et al. (1999).

For immunofluorescence analysis, Aplysia sensory neurons were cultured as described previously (Schacher and Proshansky, 1983; Chin et al., 2002). Cultures were treated with five 5 min pulses of either vehicle (L15:ASW) or $50 \mu \mathrm{M} 5-\mathrm{HT}$ with an interpulse interval of $20 \mathrm{~min}$. At various time points after the end of treatment, cells were fixed in a solution of $4 \%$ paraformaldehyde in PBS containing 30\% sucrose and blocked for $30 \mathrm{~min}$ at room temperature in Superblock blocking buffer (Pierce, Holmdel, NJ)/0.2\% Triton X-100/3\% normal goat serum. Subsequently, fixed cells were incubated overnight at $4^{\circ} \mathrm{C}$ with anti-tCREB1 antibody (1:500) or anti-pCREB1 antibody (1:500). Secondary antibody (goat anti-rabbit secondary antibody conjugated to Cy-3; 1:200 dilution; Jackson ImmunoResearch, West Grove, PA) was applied for $1 \mathrm{~h}$ at room temperature. Images were obtained with a Bio-Rad (Hercules, CA) 1024 MP confocal microscope using a $60 \times$ oil immersion lens. A z-series of optical sections through the cell body $(0.5 \mu \mathrm{m}$ increments $)$ was taken, and the section through the middle of the nucleus was used for analysis of mean fluorescence intensity with the MetaMorph Offline software (Universal Imaging Corporation, West Chester, PA). Five to 10 neurons on each coverslip were analyzed, and measurements from neurons on the same coverslip were averaged.

For the experiments that examined levels of total CREB1 in the nucleus after blocking cytoplasmic CREB1, the anti-tCREB1 antibody or purified rabbit IgG (control) (both at $0.5 \mu \mathrm{g} / \mu \mathrm{l}$ ) was injected into the cytoplasm of sensory neurons immediately after 5-HT treatment. Dextran- conjugated fluorescein $(70 \mathrm{kDa})$ was included in the injection buffer to monitor the efficiency and locus of injection. Two hours later, cells were processed for immunofluorescence analysis using anti-tCREB1 antibody (the same antibody used for injections) as primary antibody, followed by Cy-3-conjugated secondary antibody.

For the experiments that examined levels of initial phosphorylation of CREB1 in the nucleus after blocking cytoplasmic CREB1, anti-tCREB1 antibody or purified rabbit IgG was injected into the cytoplasm of sensory neurons $2 \mathrm{~h}$ before treatment with 5 -HT or vehicle. Immediately after the end of treatment, cells were fixed and processed for immunofluorescence analysis using anti-pCREB1 antibody as primary antibody, followed by Cy-3-conjugated secondary antibody.

Sensory neurons were imaged through confocal microscopy using the blue and green lines of a Kr/Ar laser in sequential mode at the minimal required power for excitation of fluorescein (injection dye) and $\mathrm{Cy}-3$ (immunoreactivity for total CREB1). Cells were excluded from further analysis if fluorescein signal was detected in the nucleus. In antibodyinjected cells, only Cy-3 immunofluorescence in the cell nucleus was analyzed. For quantification of nuclear total CREB1 staining, the nuclear membrane was first identified using the distribution of fluorescein. Because antibodies do not cross the nuclear membrane (Ohno et al., 1998), nuclear immunofluorescence is a measure of endogenous nuclear CREB1. Immunofluorescence analysis was performed in a "blind" manner.

Oligonucleotide injection. CRE (5'-GGCCTCCTTGGCTGACGTCAGAGAGAGAGTTCTGCA-3') or mutated (control) (5'-GGCCTCCTTGGCCTTAAGTGGAGAGAGAGTTCTGCA-3') oligonucleotides (IDT, San Jose, CA) were dissolved in $10 \mathrm{~mm}$ Tris, pH 7.3, $100 \mathrm{~mm} \mathrm{KCl,} \%$ dextran-conjugated Alexa $488(10 \mathrm{kDa})$ to a final concentration of 5 $\mu \mathrm{g} / \mathrm{ml}$. Oligonucleotide sequences were obtained from Dash et al. (1990). Nucleotides in bold correspond to the CRE sequence or its mutated control. Oligonucleotides were pressure injected into sensory neurons immediately after the end of treatment with either five pulses of 5-HT or vehicle. Five hours later, cells were processed for immunofluorescence using anti-tCREB1 antibody, followed by Cy-3-conjugated secondary antibody. The experimenter performing the injections and subsequent immunofluorescence analysis was "blind" to the identity of group treatment.

CRE-enhanced green fluorescent protein reporter. A CRE-enhanced green fluorescent protein (EGFP) reporter vector was prepared from the previously used CRE-lacZ vector (Kaang et al., 1993). To monitor CREdependent transcription in individual cultured sensory neurons, the lac $Z$ gene was excised from the vector with HindIII and BamHI restriction enzymes (Promega, Madison, WI) and then replaced with the EGFP gene cloned from the pNEX3-EGFP vector (gift from B. Kaang, Seoul National University, Seoul, South Korea). Cultured sensory neurons were treated with either 5-HT or vehicle as described above. Either 2 or $12 \mathrm{~h}$ after the end of the treatment, neurons were injected with the CRE-EGFP expression vector $(1 \mu \mathrm{g} / \mu \mathrm{l})$ in injection buffer containing dextran-conjugated Texas Red fluorescence marker $(70 \mathrm{kDa})$ to monitor the efficiency and locus of injection. Five hours after vector injection (i.e., either 7 or $17 \mathrm{~h}$ after the end of the treatment), cells were fixed with paraformaldehyde and processed for immunofluorescence analysis. Confocal optical sections through the middle of the nucleus were obtained and analyzed for mean fluorescence intensity. EGFP signal in the cytoplasm and red fluorescence from injection dye in the nucleus were determined by tracing the outline of the cell body and nucleus. Mean fluorescence intensity for EGFP in the cytoplasm was normalized to the amount of plasmid injected, indicated by the intensity of injected fluorescent dye in the nucleus. The normalized EGFP expression in 5-HT-treated cells was compared with that from vehicle-treated cells.

Electrophysiology. Sensorimotor cocultures were prepared as described previously (Angers et al., 2002). EPSPs were recorded from motor neurons with $10-15 \mathrm{M} \Omega$ sharp electrodes filled with $3 \mathrm{M}$ potassium acetate. Neurons with resting membrane potential more positive than $-40 \mathrm{mV}$ and input resistance $<10 \mathrm{M} \Omega$ were excluded. Stimulation of presynaptic sensory neurons was performed extracellularly using a blunt patch electrode filled with modified L15-ASW. For statistical analysis, the amplitude of the EPSP was measured during the pretest and $24 \mathrm{~h}$ after exposure 
A. CREB1 mRNA

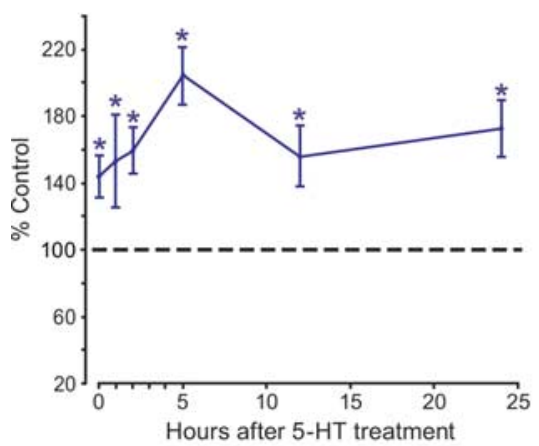

B. Total CREB1 protein

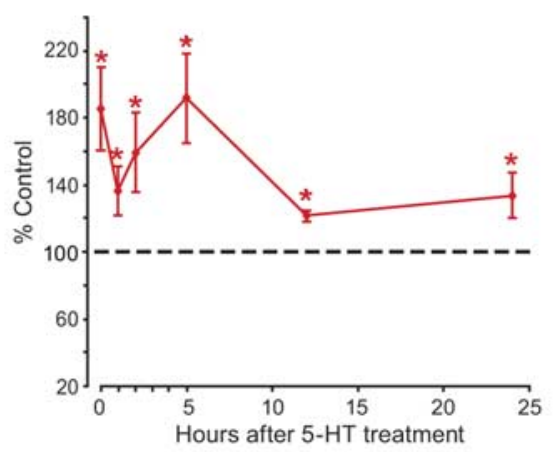

C. Phosphorylated CREB1 protein

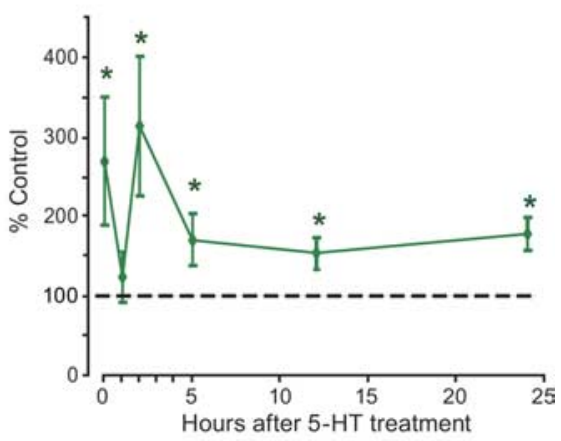

D1. Anti-total CREB1 antibody staining $2 \mathrm{~h}$ post 5-HT

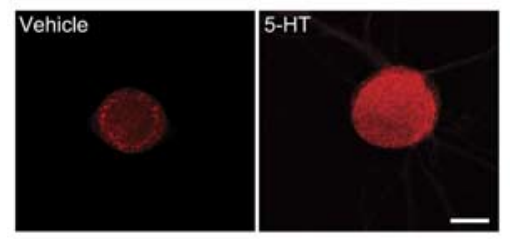

D2. CREB1 intensity $2 \mathrm{~h}$ post $5-\mathrm{HT}$

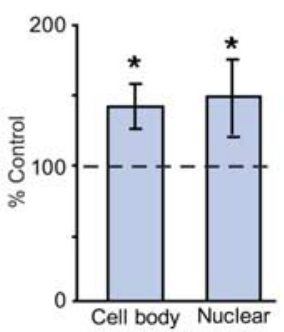

E1. CRE-EGFP expression

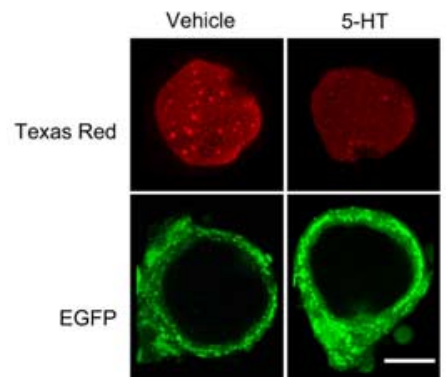

E2. EGFP intensity

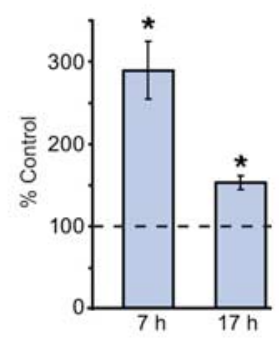

Figure 1. Dynamics of CREB1 mRNA and protein, CREB1 phosphorylation, and CRE-dependent gene transcription after 5-HT treatment. $\boldsymbol{A}$, Summary data from qRT-PCR. CREB1 mRNA was rapidly and persistently elevated for at least $24 \mathrm{~h}$ after treatment with 5 - $\mathrm{HT}$ that produces long-term facilitation. $\boldsymbol{B}$, Total CREB1 protein levels were also persistently elevated for at least $24 \mathrm{~h}$ after 5 - $\mathrm{HT}$ treatment. C, Dynamic changes of phosphorylated CREB1 after 5-HT treatment. D1, D2, Immunofluorescence staining for total-CREB1. D1, Immunoreactivity for total CREB1 in sensory neurons $2 \mathrm{~h}$ after vehicle or 5-HT treatment. Scale bar, $20 \mu \mathrm{m} . \mathbf{D 2}$, CREB1 immunoreactivity increased in both cell body and nucleus $2 \mathrm{~h}$ after 5 -HT treatment, compared with vehicle-treated control $\left({ }^{*} p<0.05\right)$. E1, E2, CRE-mediated EGFP expression is enhanced after five pulses of 5-HT for at least up to $12 \mathrm{~h}$. E1, Representative images of cells injected $12 \mathrm{~h}$ after 5 -HT or vehicle and fixed $5 \mathrm{~h}$ later. Texas Red-dextran was added to the injection buffer to confirm nuclear injection. Scale bar, $20 \mu \mathrm{m}$. E2, EGFP expression was increased in neurons injected either 2 or $12 \mathrm{~h}$ after 5 -HT treatment, compared with controls $\left({ }^{*} p<0.05\right)$.

to 5-HT or vehicle (post-test), and the post/pre ratio was formed. Data acquisition was performed using pClamp (Molecular Devices, Union City, CA). Purified anti-tCREB1 antibody $(0.5 \mu \mathrm{g} / \mu \mathrm{l})$ or rabbit IgG $(0.5$ $\mu \mathrm{g} / \mu \mathrm{l})$ in injection buffer ( $100 \mathrm{~mm} \mathrm{KCl}, 0.1 \%$ Alexa $488-10 \mathrm{kDa}$ dextran) was pressure injected in the cytoplasm of sensory neurons $1 \mathrm{~h}$ after the end of 5-HT treatment using the Eppendorf (Westbury, NY) microinjection system. The efficiency of the injection was monitored with a fluorescence microscope. The experimenter performing the electrophysiological testing was "blind" to the identity of the antibody injected. After the end of the $24 \mathrm{~h}$ posttest, a subset of cocultures injected with antiCREB1 or IgG antibody was fixed with $4 \%$ paraformaldehyde, permeabilized with $0.2 \%$ Triton X-100 in blocking buffer for $30 \mathrm{~min}$, and incubated with goat anti-rabbit secondary antibody conjugated to Cy-3 to visualize the intracellular localization of the injected primary antibody.

\section{Results}

CREB1 mRNA and protein levels in ganglia are increased for at least $24 \mathrm{~h}$ after induction of LTF

To quantify the dynamic changes in levels of CREB1 mRNA and protein, we used qRT-PCR and Western blots. After treatment of paired pleural-pedal ganglia with five pulses of either vehicle or 5 -HT, pleural ganglia were collected and frozen either immediately or $1,2,5,12$, or $24 \mathrm{~h}$ after the last treatment pulse. Compared with time-alone matched controls, CREB1 mRNA levels were significantly increased at all time points investigated (Fig. $1 A$ ) (mean percentage control \pm SEM: immediately, $144 \pm 12.7$, $t_{13}=2.8, p<0.05 ; 1 \mathrm{~h}, 152.9 \pm 27.7, t_{13}=2.7, p<0.05 ; 2 \mathrm{~h}$, $159.3 \pm 14.1, t_{13}=4.3, p<0.001 ; 5 \mathrm{~h}, 204.2 \pm 16.9, t_{13}=4.3, p<$
$0.001 ; 12 \mathrm{~h}, 155.5 \pm 17.7, t_{6}=4.7, p<0.01 ; 24 \mathrm{~h}, 172.6 \pm 17.0, t_{6}$ $=5.7, p<0.01$ ).

Next, Western blot analyses of lysates from pleural-pedal ganglia were performed to determine whether the observed changes in mRNA were associated with changes in protein levels (Fig. 1) (Fig. S1 A, available at www.jneurosci.org as supplemental material). Compared with time-alone matched controls, total CREB1 protein levels from pleural-pedal ganglia were also significantly increased at all time points investigated (Fig. $1 B$ ) (mean percentage control \pm SEM: immediately, $185.0 \pm 24.9, t_{5}=$ $4.468, p<0.01 ; 1 \mathrm{~h}, 136.3 \pm 14.8, t_{5}=7.681, p<0.001 ; 2 \mathrm{~h}$, $169.3 \pm 19.9, t_{7}=3.448, p<0.05 ; 5 \mathrm{~h}, 184.4 \pm 23.3, t_{7}=$ $3.879, p<0.01 ; 12 \mathrm{~h}, 121.4 .5 \pm 3.5, t_{4}=3.322, p<0.05 ; 24 \mathrm{~h}$, $\left.133.6 \pm 15.0, t_{4}=2.170, p<0.05\right)$. These results indicate that CREB1 itself is one of the proteins with increased expression that is associated with LTF, and that these changes occur rapidly and persist for at least $24 \mathrm{~h}$.

\section{Phosphorylation of CREB1 in ganglia is enhanced for at least $24 \mathrm{~h}$ after 5-HT treatment}

5-HT-induced increase in CREB1 expression lasts for at least 24 h. However, for a CREB1 feedback loop to be functional, CREB1 needs to be phosphorylated. Therefore, we examined the dynamics of 5-HT-induced CREB1 phosphorylation at Ser-85 (equivalent to Ser-133 in vertebrate CREB) using a phosphospecific antibody (Mohamed et al., 2005). Compared with timealone matched controls, phospho-CREB1 (pCREB1) protein levels were significantly elevated for all but one time point (Fig. 1C) 
(mean percentage control \pm SEM: immediately, $269.6 \pm 80.4, t_{5}$ $=3.573, p<0.05 ; 1 \mathrm{~h}, 123.6 \pm 31.8, t_{5}=1.498, p=0.104 ; 2 \mathrm{~h}$, $313.6 \pm 88.6, t_{5}=6.132, p<0.01 ; 5 \mathrm{~h}, 170.4 \pm 32.9, t_{5}=$ $2.407, p<0.05 ; 12 \mathrm{~h}, 153.2 \pm 20.5, t_{4}=3.664, p<0.05 ; 24 \mathrm{~h}$, $\left.178 \pm 21.2, t_{5}=4.043, p<0.01\right)$. The dynamics of CREB1 phosphorylation after LTF induction appeared complex (Fig. 1C) (Fig. S1 B, available at www.jneurosci.org as supplemental material). An initial peak was followed by a dramatic drop, a subsequent rapid recovery back to high levels, and a final drop to sustained phosphorylation level at $\sim 150 \%$ of controls. The mechanisms and the significance of these dynamics are unclear. However, the results indicate that phosphorylation of CREB1 is enhanced for a prolonged period after treatment and therefore CREB1 could participate in a positive feedback loop.

\section{Total and phosphorylated CREB1 levels are increased in sensory neurons after 5-HT treatment}

To confirm that the observed increase of both total and phosphorylated CREB1 in the ganglia is also present in the subpopulation of SNs, which are the presynaptic sites of plasticity, we examined levels of CREB1 protein in cultured SNs using immunofluorescence and confocal microscopy. Two hours after the end of treatment with 5-HT, total CREB1 protein levels were increased in both cell body and nucleus. Compared with vehicle-treated controls, 5-HT-treated cells showed a $44 \pm$ $16 \%$ increase in tCREB 1 immunofluorescence in the cell body and a $49 \pm 24 \%$ increase in the nucleus (Fig. 1D) (cell body staining, $t_{3}=3.515, p<0.05$; nuclear staining, $t_{3}=3.323, p<$ 0.05 ; representing a total of 23 vehicle-treated neurons and 24 5-HT-treated neurons). These results indicate that the changes in CREB1 protein measured in ganglia are recapitulated in SNs. Moreover, not only are levels of total CREB1 elevated, but nuclear levels of phospho-CREB1 are also elevated (mean percentage control \pm SEM: $123.2 \pm 6, t_{3}=3.182$, $p<0.05$, representing a total of 26 vehicle-treated neurons and 27 5-HT-treated neurons) (Fig. S2, available at www. jneurosci.org as supplemental material). Levels of phosphoCREB1 in the cytoplasm and processes were extremely low compared with nuclear levels, indicating that CREB1 phosphorylation is primarily a nuclear event.

\section{A CREB1 feedback loop regulates expression of CREB1 after 5-HT treatment}

What mechanisms regulate the increased expression of CREB1 after LTF induction? Previous work showed that CREB1 can bind to the CRE in its own promoter region and that this binding is increased after treatment with 5-HT (Mohamed et al., 2005). To examine whether the enhancement of CREB1 expression after 5-HT is regulated by CREB1 itself, we injected mutated (control) or CRE oligonucleotides in sensory neurons immediately after treatment with 5 -HT or vehicle and examined the effect on CREB1 expression $5 \mathrm{~h}$ later through immunofluorescence analysis. The CRE oligonucleotides injected in excess should compete with endogenous sites for CREB1 binding and therefore block CREB1-dependent transcription. Results indicated that injection of CRE oligonucleotides blocked the 5-HT-induced increase in CREB1 expression [normalized to control group (mutated plus vehicle): CRE plus 5-HT, $93.33 \pm 7.72 \%$; mutated plus 5-HT, $122.02 \pm$ 5.66\%; CRE plus vehicle, $103.09 \pm 4.85 \%$ ]. One-way ANOVA indicated a significant difference between the three groups $\left(F_{(2,18)}=6.503 ; p<0.05\right)$. Subsequent pair-wise comparisons using Student-Newman-Keuls post hoc tests indicated that CRE oligonucleotides blocked 5-HT-induced increase in CREB1 (mutated plus 5-HT vs CRE plus 5-HT, $q=3.51 ; p<$ 0.05 ) (Fig. S3, available at www.jneurosci.org as supplemental material). These results provide direct evidence that CREB1 drives its own expression and support the existence of a CREB1-positive feedback loop during consolidation of longterm facilitation.

\section{CRE-dependent gene transcription is increased at least up to $12 \mathrm{~h}$ after 5-HT treatment}

What is the function of increased CREB1 expression? We reasoned that if total and phosphorylated CREB1 levels are persistently elevated after 5-HT treatment, CRE-mediated transcription should be elevated as well. To directly measure CREmediated transcription, we injected a CRE-EGFP reporter construct in cultured SNs 2 or $12 \mathrm{~h}$ after exposure to five $5 \mathrm{~min}$ pulses of either 5-HT or vehicle. Five hours after injection (corresponding to either 7 or $17 \mathrm{~h}$ after the end of treatment), cells were fixed and analyzed for EGFP expression. Compared with vehicle-treated cells, 5-HT-treated cells showed increased EGFP expression at both $7 \mathrm{~h}$ (Fig. S4, available at www.jneurosci.org as supplemental material) and $17 \mathrm{~h}$ (Fig. $1 \mathrm{E}$ ) (mean percentage control \pm SEM: $7 \mathrm{~h}, 287.77 \pm 35.14, t_{3}=4.51, p<0.05 ; 17 \mathrm{~h}$, $\left.151.72 \pm 8.74, t_{3}=4.14, p<0.05\right)$. These results agree with the persistent elevation and phosphorylation of CREB1 and provide direct evidence that CRE-mediated transcription is not limited to the induction phase of LTF.

\section{Sequestering cytoplasmic, including newly synthesized, CREB1 after 5-HT treatment attenuates LTF}

To further investigate the functional role of increased CREB1 and the CREB1 feedback loop in LTF, we injected anti-tCREB1 antibody in the cytoplasm of SNs after the end of 5-HT treatment and examined the effect on $24 \mathrm{~h}$ LTF. Briefly, after assessment of basal synaptic strength, sensorimotor cocultures were treated with five pulses of vehicle or 5-HT. One hour after the end of treatment, anti-tCREB1 antibody or purified IgG (control) was injected into the sensory neuron. A fluorescent dye (Alexa 488-dextran; $10 \mathrm{kDa}$ ) was coinjected to help determine the locus and efficiency of injections (Fig. 2A2). The extent of LTF was assessed $24 \mathrm{~h}$ after 5-HT, during the post-test (Fig. 2A3,A4). One-way ANOVA indicated a significant difference among the four groups (mean percentage post/pre \pm SEM: IgG-alone, 86.1 $\pm 5.6, n=3$; IgG plus $5-\mathrm{HT}$, $142.3 \pm 12.5, n=7$; anti-tCREB1-alone, $78.3 \pm 14.4, n=3$; anti-tCREB1 plus 5-HT, $103.0 \pm 5.7, n=4 ; F_{(3,16)}=6.3 ; p<$ $0.01)$. Subsequent pair-wise comparisons using StudentNewman-Keuls post hoc tests indicated that 5-HT induced significant facilitation in the IgG-injected cocultures $(q=$ $4.53 ; p<0.05)$ but not in the anti-tCREB1-injected cocultures $(q=1.8 ; p>0.05)$. Moreover, the IgG plus 5 -HT group was significantly different from the anti-tCREB1 plus 5-HT group $(q=3.49 ; p<0.05)$. These results suggest that disruption of the CREB1 feedback loop through sequestration of cytoplasmic, including newly synthesized, CREB1 impaired LTF. Injection of IgG or anti-tCREB1 antibody alone did not significantly affect synaptic transmission over a $24 \mathrm{~h}$ period $(q=$ $0.53 ; p>0.05$ ) (Fig. 2A4). Therefore, blocking cytoplasmic, including newly synthesized, CREB1 through antibody injection significantly impaired LTF without affecting basal transmission.

The interpretation of the electrophysiology experiment de- 


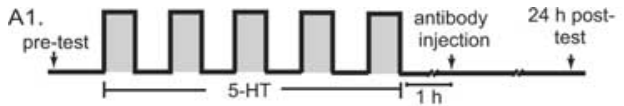

A2. Injected anti-tCREB1 antibody staining

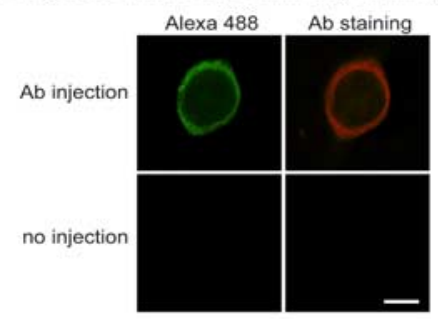

B1.

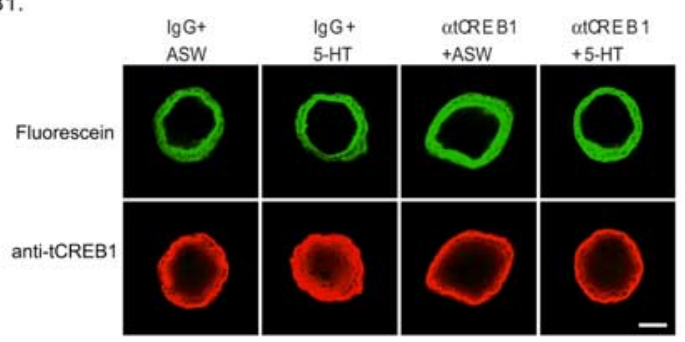

A3.

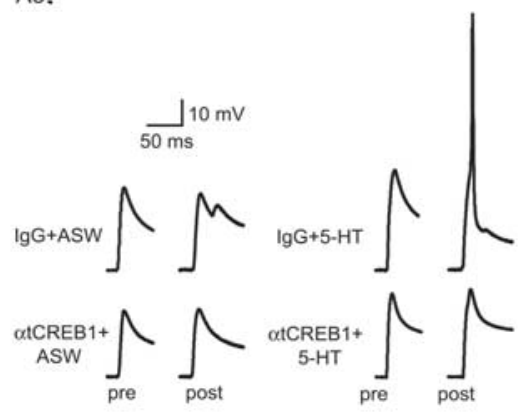

B2. Nuclear CREB1 intensity

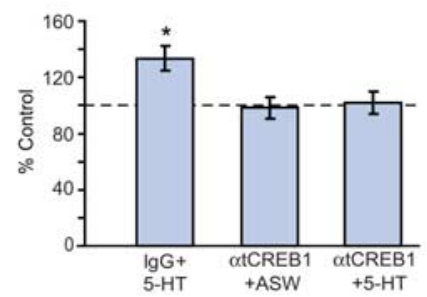

A4. Synaptic strength
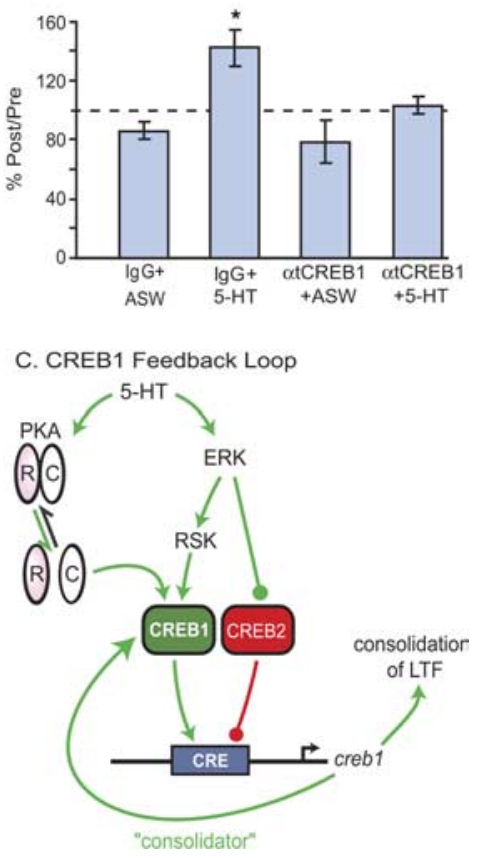

Figure 2. Blocking cytoplasmic, including newly synthesized, CREB1 impaired LTF and associated increase in nuclear CREB1. A1, Protocol for control lgG or anti-tCREB1 antibody injection and electrophysiological testing. Antibodies were injected in the cytosol of sensory neurons $1 \mathrm{~h}$ after the end of treatment with 5-HT. A2, Confocal images of a neuron injected with both anti-tCREB1 antibody and Alexa 488-dextran and of a noninjected neuron. Neurons were incubated with secondary antibody only. In the injected neuron, tCREB1 immunoreactivity was limited to the cytoplasm, suggesting that the antibody did not cross the nuclear membrane. Minimal autofluorescence and background staining were observed in the noninjected neuron. Scale bar, $20 \mu \mathrm{m}$. $\mathbf{A 3}$, EPSPs recorded from sensorimotor cocultures before (pre) and $24 \mathrm{~h}$ after (post) treatment with 5 -HT or vehicle. In some experiments, the facilitation was so large that it led to the initiation of a spike. In these cases, the EPSP was assigned a value of $65 \mathrm{mV}$ as was done in previous studies (Zhao et al., 2003). A4, Summary data. One-way ANOVA followed by post hoc tests showed that injection of anti-tCREB1 antibody blocked LTF without significantly affecting basal transmission over $24 \mathrm{~h}$. B1, Cytoplasmic injection of anti-tCREB1 antibody blocked 5-HT-induced increase in nuclear tCREB1. In the top panel of representative images, the cytoplasmic locus of fluorescein indicated antibodies were injected into the cytoplasm. In the bottom panel, tCREB1 levels in the nucleus $2 \mathrm{~h}$ after 5 - $\mathrm{HT}$ treatment was measured by immunofluorescence staining using anti-tCREB1 antibody. Scale bar, $20 \mu \mathrm{m}$. B2, Anti-tCREB1 injection blocked 5-HT-enhanced expression of tCREB1 in nucleus ( $n=$ 4 independent experiments, representing 22 lgG-injected plus vehicle, 21 lgG-injected plus 5-HT, 21 anti-tCREB1-injected plus vehicle, and 23 anti-tCREB1-injected plus 5-HT neurons). C, Model of CREB1 feedback loop for the induction and consolidation of long-term memory in Aplysia (see Discussion for description).

scribed above relies on three assumptions pertaining to the cytoplasmic injection of anti-tCREB1 antibody: (1) antibody injection blocks the CREB1 feedback loop; (2) antibody injection does not affect resting levels of nuclear CREB1; and (3) antibody injection does not interfere with the regulation of preexisting nuclear CREB1. We performed experiments to test each of these assumptions. We first examined whether injection of anti-tCREB1 antibody disrupted the CREB1 feedback loop. With the loop intact, treatment with 5-HT should result in increased levels of CREB1 in the nucleus (Fig. 1D). We therefore injected SNs with either control IgG or anti-tCREB1 antibody immediately after the end of treatment with either 5-HT or vehicle, and we assayed levels of CREB1 in the nucleus $2 \mathrm{~h}$ later. Results showed that injection of anti-tCREB1 antibody blocked 5-HT-induced increase in nuclear CREB1 (normalized to IgG plus vehicle: anti-tCREB1 plus 5-HT, $101.9 \pm$ 7.9\%; IgG plus 5-HT, $133.5 \pm 8.8 \%$; anti-tCREB1 plus vehicle, $98.3 \pm 7.6 \%$ ) (Fig. 2 B). One-way ANOVA indicated a significant difference among these three groups $\left(F_{(2,11)}=17.144\right.$; $p<0.01)$. Subsequent pair-wise comparisons using StudentNewman-Keuls post hoc tests indicated that anti-tCREB1 antibody injection blocked the 5-HT-induced increase in nuclear CREB1 (anti-tCREB1 plus 5-HT vs IgG plus 5-HT, $q=6.204$; $p<0.05)$. These results suggest that cytoplasmic injection of anti-tCREB1 antibody after 5-HT treatment disrupts the CREB1-positive feedback loop.
To test the second assumption that injection of anti-tCREB1 antibody did not affect resting levels of nuclear CREB1, we injected anti-tCREB1 antibody or IgG in the cytoplasm of sensory neurons and assayed levels of nuclear CREB1 $24 \mathrm{~h}$ after injection. It is possible to measure nuclear CREB1 using the same antibody for immunostaining as used for cytoplasmic injection, because the anti-tCREB1 antibody injected into the cytoplasm did not enter the nucleus. This was indicated by the lack of nuclear CREB1 staining in cells injected with anti-tCREB1 antibody in the cytoplasm, fixed and permeabilized $24 \mathrm{~h}$ later, and stained with a secondary antibody only (Fig. $2 A 2)(n=4)$. When nuclear levels of CREB1 were measured $24 \mathrm{~h}$ after cytoplasmic injection of anti-tCREB1 antibody, we found no difference in nuclear CREB1 levels between anti-tCREB1-injected and IgG-injected cells (in arbitrary units: IgG, $112.1 \pm 7.4$; anti-tCREB1, $111.3 \pm$ $10.8 ; n=3$ experiments encompassing 45 cells; $t_{2}=0.293 ; p>$ $0.05)$. Therefore, antibody injection does not affect resting levels of nuclear CREB1.

To examine the third assumption that anti-tCREB1 antibody injection does not interfere with the regulation of preexisting nuclear CREB1, we injected anti-tCREB1 antibody in SNs $2 \mathrm{~h}$ before treatment with either vehicle or 5-HT and measured levels of nuclear pCREB1 immediately after treatment. We found that 5-HT induced an increase in levels of nuclear pCREB1 despite the antibody injection (normalized to anti-tCREB1 plus vehicle: anti-tCREB1 plus 5-HT, $118.7 \pm$ 
$5 \% ; t_{3}=4.432 ; p<0.05$, representing a total of 34 neurons). Thus, injection of anti-tCREB1 antibody does not appear to interfere with the regulation of preexisting nuclear CREB1. Furthermore, these results indicate that initial CREB1 phosphorylation at Ser-85 is not sufficient for LTF and corroborate the requirement for a CREB1-positive feedback loop in the consolidation of LTF.

\section{Discussion}

A critical role for CREB1 in the induction of long-term synaptic plasticity was demonstrated previously (Dash et al., 1990; Bartsch et al., 1998). Dash et al. (1990) found that injecting CRE oligonucleotides to sequester and therefore functionally block endogenous CREB1 before 5-HT treatment blocked LTF. Subsequent studies by Bartsch et al. (1998) found that injection of antiCREB1 antibodies before induction of LTF blocked facilitation at $24 \mathrm{~h}$. Since that time, numerous studies in invertebrates and vertebrates have implicated CREB in long-term synaptic plasticity (for review, see Lonze and Ginty, 2002). However, these previous experiments do not permit differentiation between a role of CREB in induction and consolidation of synaptic plasticity. By manipulating levels of CREB1 after induction, we found that CREB1 has a specific role in the consolidation phase of long-term plasticity.

Our results showed that a 5 -HT treatment protocol that produces LTF led to increased levels of CREB1 mRNA both immediately and up to $24 \mathrm{~h}$ after 5-HT treatment. Moreover, this increased message was associated with increased expression of total and phosphorylated CREB1 protein. As suggested by previous work in our laboratory (Mohamed et al., 2005), we found that the increase in total CREB1 is regulated by CREB1 itself, corroborating the existence of a CREB1-positive feedback loop. Could this loop drive CRE-dependent transcription for several hours after LTF induction? Little is known about transcription beyond the induction phase of LTF. Although initial experiments suggested that transcription is required only during LTF induction (Montarolo et al., 1986), later studies supported a role for CCAAT enhancer-binding protein (C/EBP)-mediated transcription up to $9 \mathrm{~h}$ after $5-\mathrm{HT}$ for LTF measured at $24 \mathrm{~h}$ (Alberini et al., 1994). Our data support the presence of increased CRE-mediated transcription for at least $12 \mathrm{~h}$ after 5 -HT (Fig. $1 E$ ). This result suggests that either ongoing transcription beyond $9 \mathrm{~h}$ is necessary for the consolidation of LTF but does not depend on C/EBP, or that this late transcription is required for late-LTF, which is observed at 48 and $72 \mathrm{~h}$ after 5-HT (Zhang et al., 1997; Casadio et al., 1999).

The results of our experiments on the CREB1 feedback loop and its role in consolidation of LTF give rise to the following model (Fig. 2C): treatment with 5-HT results in enhanced recruitment of phosphorylated CREB1 to its promoter region and a concomitant decrease in the binding of the transcriptional repressor CREB2 to the CREB1 promoter region (Mohamed et al., 2005). These changes in binding would lead to the sustained increase in CREB1 expression. In turn, the increase in CREB1 levels and its persistent phosphorylation, probably via the persistent activation of PKA (Müller and Carew, 1998), would lead to further creb1 expression and thereby close the feedback loop, leading to prolonged expression of CREB1. Increased expression of CREB1 would not only lead to activation of creb1 but also of other genes that have CREs in their promoters, thus regulating the synthesis of proteins necessary for the consolidation of LTF.
The dynamics of CREB expression could explain at least in part why multiple spaced learning trials are more effective in producing long-term memory than single trials or even multiple trials when massed together (i.e., massed training) (Frost et al., 1985; Wainwright et al., 2002). Indeed, in Aplysia, four multiple trials (five shocks per trial) separated by $24 \mathrm{~h}$ intervals produce memory (sensitization) that lasts for $>7 \mathrm{~d}$, whereas a single trial produces memory that lasts for only $4 \mathrm{~d}$ (Frost et al., 1985). Our results indicate that a second trial $24 \mathrm{~h}$ after the first would occur at a time associated with "residual" elevation of CREB1 levels. Consequently, more CREB1 would be available to activate CRE-mediated gene expression, "boosting" gene expression above that produced by the first training trial.

An extension of this line of reasoning is that the dynamics of CREB expression could help inform the development of optimal behavioral training protocols. An understanding of the dynamics of the molecular cascades involved in the induction and consolidation of memory can be used to develop training procedures that are "in phase" with those cascades, thereby leading to enhanced learning and memory.

\section{References}

Alberini CM, Ghirardi M, Metz R, Kandel ER (1994) C/EBP is an immediate-early gene required for the consolidation of long-term facilitation in Aplysia. Cell 76:1099-1114.

Angers A, Fioravante D, Chin J, Cleary LJ, Bean AJ, Byrne JH (2002) Serotonin stimulates phosphorylation of Aplysia synapsin and alters its subcellular distribution in sensory neurons. J Neurosci 22:5412-5422.

Antzoulatos EG, Cleary LJ, Eskin A, Baxter DA, Byrne JH (2003) Desensitization of postsynaptic glutamate receptors contributes to high-frequency homosynaptic depression of Aplysia sensorimotor connections. Learn Mem 10:309-313.

Bartsch D, Casadio A, Karl KA, Serodio P, Kandel ER (1998) CREB1 encodes a nuclear activator, a repressor, and a cytoplasmic modulator that form a regulatory unit critical for long-term facilitation. Cell 95:211-223.

Casadio A, Martin KC, Giustetto M, Zhu H, Chen M, Bartsch D, Bailey CH, Kandel ER (1999) A transient, neuron-wide form of CREB-mediated long-term facilitation can be stabilized at specific synapses by local protein synthesis. Cell 99:221-237.

Chin J, Angers A, Cleary LJ, Eskin A, Byrne JH (1999) TGF-betal in Aplysia: role in long-term changes in the excitability of sensory neurons and distribution of TbetaR-II-like immunoreactivity. Learn Mem 6:317-330

Chin J, Angers A, Cleary LJ, Eskin A, Byrne JH (2002) Transforming growth factor $\beta 1$ alters synapsin distribution and modulates synaptic depression in Aplysia. J Neurosci 22:RC220(1-6).

Dash PK, Hochner B, Kandel ER (1990) Injection of the cAMP-responsive element into the nucleus of Aplysia sensory neurons blocks long-term facilitation. Nature 345:718-721.

Frost WN, Castellucci VF, Hawkins RD, Kandel ER (1985) Monosynaptic connections made by the sensory neurons of the gill- and siphonwithdrawal reflex in Aplysia participate in the storage of long-term memory for sensitization. Proc Natl Acad Sci USA 82:8266-8269.

Kaang BK, Kandel ER, Grant SG (1993) Activation of cAMP-responsive genes by stimuli that produce long-term facilitation in Aplysia sensory neurons. Neuron 10:427-435.

Levenson J, Byrne JH, Eskin A (1999) Levels of serotonin in the hemolymph of Aplysia are modulated by light/dark cycles and sensitization training. J Neurosci 19:8094-8103.

Lonze BE, Ginty DD (2002) Function and regulation of CREB family transcription factors in the nervous system. Neuron 35:605-623.

Marinesco S, Carew TJ (2002) Serotonin release evoked by tail nerve stimulation in the CNS of Aplysia: characterization and relationship to heterosynaptic plasticity. J Neurosci 22:2299-2312.

Mauelshagen J, Sherff CM, Carew TJ (1998) Differential induction of long-term synaptic facilitation by spaced and massed applications of 
serotonin at sensory neuron synapses of Aplysia californica. Learn Mem 5:246-256.

Mohamed HA, Yao W, Fioravante D, Smolen PD, Byrne JH (2005) cAMPresponse elements in Aplysia creb1, creb2, and Ap-uch promoters: implications for feedback loops modulating long term memory. J Biol Chem 280:27035-27043.

Montarolo PG, Goelet P, Castellucci VF, Morgan J, Kandel ER, Schacher S (1986) A critical period for macromolecular synthesis in long-term heterosynaptic facilitation in Aplysia. Science 234:1249-1254.

Müller U, Carew TJ (1998) Serotonin induces temporally and mechanistically distinct phases of persistent PKA activity in Aplysia sensory neurons. Neuron 21:1423-1434.

Ohno M, Fornerod M, Mattaj IW (1998) Nucleocytoplasmic transport: the last 200 nanometers. Cell 92:327-336.

O’Leary FA, Byrne JH, Cleary LJ (1995) Long-term structural remodeling in
Aplysia sensory neurons requires de novo protein synthesis during a critical time period. J Neurosci 15:3519-3525.

Schacher S, Proshansky E (1983) Neurite regeneration by Aplysia neurons in dissociated cell culture: modulation by Aplysia hemolymph and the presence of the initial axonal segment. J Neurosci 3:2403-2413.

Wainwright ML, Zhang H, Byrne JH, Cleary LJ (2002) Localized neuronal outgrowth induced by long-term sensitization training in Aplysia. J Neurosci 22:4132-4141.

Zhang F, Endo S, Cleary LJ, Eskin A, Byrne JH (1997) Role of transforming growth factor-beta in long-term synaptic facilitation in Aplysia. Science 275:1318-1320.

Zhao Y, Hegde AN, Martin KC (2003) The ubiquitin proteasome system functions as an inhibitory constraint on synaptic strengthening. Curr Biol 13:887-898. 